\title{
Modernización y consumo en Andalucía y Cataluña (1995-2006)
}

\author{
M. a Dolores Martín-Lagos López \\ Universidad de Granada. Departamento de Sociología
}

Recibido: 23-01-2008

Aceptado: 15-04-2009

\section{Resumen}

En este artículo se analizan las pautas de consumo en Andalucía y Cataluña y su evolución durante el periodo 1995-2006. Se aplica la teoría de la modernización al estudio del consumo. Se definen cinco rasgos de la modernización aplicados al consumo que se quieren contrastar: visión de desarrollo o progreso de la sociedad, extensión de ciertos objetos de consumo entre la población, aparición de nuevas necesidades, mayor igualdad social respecto a los objetos de consumo, aleatoriedad en el comportamiento o individuación y un nuevo tipo de consumidor más responsable. Para comprobar los objetivos e hipótesis formuladas se utiliza una base de datos de ASEP. El nivel de consumo se mide a través de la extensión del consumo entre la población y el número de objetos de consumo que por término medio se poseen. Los resultados muestran la aparición y extensión de objetos de consumo asociados con la tecnología entre la población. Ante este nuevo panorama se percibe más desigualdad en 2006 que en 1995. Las categorías sociales siguen explicando el consumo. El nivel educativo y el nivel de ingresos son las que más explican las diferencias aunque surgen nuevas variables explicativas como la edad y los valores individuales, que vienen a confirmar la tesis posmoderna. Se aprecian rasgos de un nuevo consumidor con valores posmaterialistas que consume menos. Esta tendencia se observa en grupos con mejor posición social de Cataluña. En Andalucía persiste la asociación entre posición social y consumo. Palabras clave: individuación, consumo, objetos materiales, consumerismo, desarrollo, tecnología.

\section{Abstract. Modernization and consumption in Andalusia and Catalonia (1995-2006)}

This article analyzes consumption patterns in Andalusia and Catalonia and their evolution during the period from 1995 to 2006 . The theory of modernization is applied to the study of consumption. Five characteristics of modernization are defined and applied to consumption and later contrasted: the vision of development or progress in the society, the dispersion of certain objects of consumption within the population, the appearance of new necessities, greater equality regarding objects of consumption, randomness in behavior or individuation and a new type of more responsible consumers. To test the objectives and hypotheses formulated an ASEP data base was used. The level of consumption was measured by the extension of consumption across the population and the number of objects of consumption associated with technology among the population. According this new prospect, there is more inequality to be observed in 2006 than in 1995 . Social categories continue to explain consumption. The level of education and level of income are the categories that most explain the differences, although new explanatory variables emerge such 
as age and individual values, which confirm the postmodern thesis. The characteristics of a new consumer with post-materialist values who consumes less can be perceived. This tendency is observed in groups with a higher social position in Catalonia. In Andalusia the association between social position and consumption persists.

Key words: individuation, consumption, material objects, consumerism, development, technology.

\section{Sumario}

$\begin{aligned} \text { 1. Objetivos y antecedentes } & \text { 4. Conclusiones } \\ \text { 2. Metodología } & \text { Referencias bibliográficas } \\ \text { 3. Resultados } & \end{aligned}$

\section{Objetivos y antecedentes}

El principal objetivo de esta investigación es analizar las pautas de consumo en Andalucía y Cataluña y su evolución durante el periodo 1995-2006. Se trata de la segunda investigación de esta naturaleza, que tiene como objetivo analizar las estructuras sociales de Andalucía y Cataluña (Gobernado, 1996). Se trata de comprender por qué unas sociedades son más modernas y están más desarrolladas que otras. Para lograr esta aproximación, es preciso delimitar teóricamente los rasgos que distinguen a las sociedades modernas en términos de consumo y, posteriormente, comparar empíricamente estos rasgos en las dos sociedades.

La teoría sociológica nos ofrece dos definiciones: el concepto clásico de modernización y la modernidad reflexiva posterior propia de la sociedad industrial actual. Hasta los años ochenta, las teorías clásicas de la modernización - tanto el concepto estructural-funcionalista como los modelos marxistas de desarrollo de los medios de comunicación - entendían la modernidad como «resultado positivo de la aplicación sistemática y masiva de la ciencia y la tecnología» (Solé, 1996: 189). Las teorías de la modernidad en una fase avanzada o tardía (Giddens, 1990) destierran el mito de la ciencia y describen una nueva sociedad moderna, más allá de su diseño industrial clásico, a la que le añaden calificativos como reflexiva, incontrolable, de riesgo o individualizada (Beck, 1992).

Tanto en el plano teórico como en el empírico, hay un primer rasgo de la modernidad: la visión evolucionista, de desarrollo o de progreso de la sociedad. «Las etapas del crecimiento económico» que publica Rostov en los años sesenta conducen desde la sociedad tradicional hacia la era del consumo de masas ${ }^{1}$.

1. Alonso y Conde (1994: 95) definen la sociedad de masas como la confluencia de las definiciones cuantitativas, según las cuales se ponen al alcance masivo y regular un conjunto amplio de bienes industriales, y las definiciones cualitativas, que definen la sociedad de consumo en términos de organización, planificación y control empresarial del proceso de demanda, de manera que, en su estadio opulento, las necesidades van siendo creadas sin 
La sociedad de consumo se corresponde con la modernidad clásica de la sociedad industrial. La fe en el progreso permitía predecir lo que ocurriría en otras sociedades, pero en la década de los años setenta las teorías evolucionistas fueron duramente criticadas. Se consideraba que las teorías de la modernidad tenían una visión desde occidente, y se proponía como "moderno y avanzado" un modelo que explotaba a los países más pobres (Wallerstein, 1979). Otras investigaciones desmontaban empíricamente el supuesto evolucionismo arguyendo que, en algunas sociedades no occidentales, la extensión de los patrones de consumo capitalista no se había producido (Hamilton, 1977).

En los últimos años, las investigaciones han vuelto a basarse en estas teorías, aunque proponiendo nuevas interpretaciones. Concretamente, Inglehart y Welzel (2006) ofrecen una «teoría de la modernización revisada». Siguen aceptando la idea de progreso de las sociedades, pero con limitaciones. Se pueden predecir los cambios que se producen en las sociedades porque están relacionados con el desarrollo socioeconómico, pero solo puede hablarse en términos de probabilidad. Las sociedades postindustriales se transforman en la misma dirección, pero las diferencias entre ellas siguen existiendo.

En sociología del consumo, Ortí (1994) distingue dos fases en el capitalismo industrial: una primera fase que tiene como modelo económico un sistema capitalista centrado en la producción y que se desarrolla de un modo importante durante el siglo XIX. Una segunda fase avanzada o madura del capitalismo industrial en el siglo XX o neocapitalismo de consumo, cuyo modelo económico se centra en el consumo de masas. Alonso (2005) desarrolla el proceso desde la formación de la norma de consumo de masas fordista, cuando se implanta el sistema de producción mercantil, se reforma el mercado de trabajo, se pone en marcha un mercado de bienes de consumo duradero y se organiza científicamente el trabajo (taylorismo), y la extensión de la norma de consumo de masas, donde se amplía el fordismo como organizador de tiempos y estilos de vida. Y, en último término, una etapa en la que el modelo anterior entra en crisis (1970) y aparece la norma de consumo postfordista, caracterizada por la fragmentación, la individualización y nuevas desigualdades. No sabemos aún cuál será la siguiente etapa tras la actual crisis económica.

Como segundo rasgo de la sociedad moderna, encontramos que el desarrollo o el progreso de la sociedad en el consumo ha sido medido mediante la extensión de ciertos objetos de consumo entre la población y la aparición de nuevas necesidades ${ }^{2}$. En 1970, Allan Schnaiberg, empleando las teorías de la modernización, hace operativo el término modernismo desglosándolo en seis dimensiones, entre las que se encuentra la de producción y consumo. Esta dimensión

cesar la producción de mercancías. Si las sociedades tradicionales se basan en la retórica de la necesidad, las sociedades modernas se caracterizan por la retórica de "querer», del deseo. Hay un contraste entre ambas sociedades, puesto que en la sociedad moderna «los consumidores esperan ser capaces de satisfacer sus deseos mientras satisfacen sus necesidades» (Campbell, 1998: 242).

2. Para ampliar el concepto de necesidad, ver Ortí, 1994. 
es medida mediante el porcentaje de propietarios de radio, de máquina de coser, de producción de jerseys, de vestidos, de sopas, de concentrados de tomate, de encurtidos y el de hogares que no elaboran alimentos de modo casero.

De la misma forma, las investigaciones empíricas llevadas a cabo en España analizan la extensión de objetos de consumo entre la población como indicador de desarrollo. A finales de los años sesenta, la mayor parte de los autores del libro España, ¿Una sociedad de consumo? (Míguez et al., 1969) coincidían en señalar que el país no podía ser definido (todavía) como una sociedad de consumo de masas. Así lo mencionaba José Castillo aunque para Amando de Miguel la tendencia era irreversible. José Félix Tezanos, en 1984, publica un artículo titulado "Cambio social y modernización en la España actual», en el que dedica un apartado al consumismo y a la sociedad de consumo. Tras su análisis de los datos (1958-1981), concluye que los españoles han visto elevado su nivel de vida objetivo de una manera significativa durante los primeros años de la transición democrática.

El excelente recorrido analítico de Alonso y Conde en la Historia del consumo en España revela que, en los años sesenta, acontece en el país un gran crecimiento cuantitativo en todas las áreas de consumo siguiendo el modelo de consumo de masas: en primer lugar, vivienda y electrodomésticos; en segundo lugar, automóviles y otros consumos (1994: 171). Esta pauta general no impide señalar que España mantiene ciertas peculiaridades en el consumo, las cuales están motivadas por un fuerte "valor simbólico de la propiedad», lo que le lleva en ocasiones a adquirir productos más por su valor de honor y distinción que por su funcionalidad.

En todo caso, la modernidad ha sido y sigue siendo la meta alcanzable, el objetivo político deseado por los gobiernos ${ }^{3}$. También la población emplea el consumo de determinados objetos materiales como indicador de que la sociedad está cambiando. Véase el trabajo de campo de Boyd Gillete (2000) en China, en el que intenta clarificar la relación entre consumo y modernización. La población, en sus discursos, considera la minifalda como símbolo de modernización, cambio y progreso. También en la España de los años setenta y ochenta los patrones de consumo de los emigrantes españoles a su regreso de Francia o Alemania reflejaban la prosperidad vivida en una sociedad más moderna.

El tercer rasgo de la modernidad es la mayor igualdad social respecto a los objetos de consumo, es decir, su extensión a un mayor abanico de la población. Junto con Alonso y Conde (1994), otros muchos autores coinciden en señalar, en las primeras etapas de la sociedad de consumo de masas en España, una tremenda dualidad social entre los que consumen y los que carecen de los servicios básicos. Y a la desigualdad social se le une una gran desigualdad territo-

3. En China, el gobierno de Deng Xiaoping (1978) prometía las «cuatro modernizaciones» en agricultura, industria, cuestiones militares y tecnología (Boyd, 2000). En el año 2001, el presidente de la Junta de Andalucía, Manuel Chaves, planteó el proyecto de segunda modernización de Andalucía, asociada a la mejora de la tecnología y su extensión entre el conjunto de la población. 
rial. Parece que la producción en masa deriva en un consumo de masas y que los objetos de consumo son un mecanismo de igualación social. Amando de Miguel discute esta aseveración. Frente a aquellos que piensan que, en la etapa de consumo de masas, unas sociedades se parecerían unas a otras, para este autor el consumo generalizado implica complejidad y diversidad (2001: 203237). «La sociedad de consumo no conduce a la pretendida homogeneización de la conducta, sino a su diversificación» (1987: 23).

El cuarto rasgo que define a la modernidad es la aleatoriedad en el comportamiento o individuación. El individuo se independiza cada vez más de las categorías sociales de origen y de pertenencia. Su comportamiento cada vez tiene menos que ver con esas categorías sociales. El consumo se explica cada vez menos por los valores religiosos (Weber, 1988 [1905]); por la posición social (Veblen, 1963 [1899]); por el hábitat rural o urbano (Simmel, 2002 [1903]); por el tipo de ocupación (Goldthorpe et al., 1968); por el habitus, medido a través de la titulación alcanzada y de la ocupación del padre (Bourdieu, 1988 [1979]), o por la edad (Castillo, 1982).

Durante la década de 1980 y 1990, las variables sociales parecen ser cada vez menos explicativas del tipo de consumo. La libertad, más allá del consumo de masas de los años cincuenta que se basaba en la uniformidad, se transmuta en heterogeneidad que fragmenta las formas y los estilos de vida de los individuos postmodernos. De la ética del trabajo, propia de la sociedad moderna, se ha pasado a la estética del consumo, propia de la modernidad líquida, en la que los consumidores frustrados se alían a comunidades volátiles, como prueba de su paradójica libertad de elección (Bauman, 1992). En la alta modernidad, los individuos buscan reflexivamente su identidad (Giddens, 1991), y los objetos de consumo son un mecanismo para ello. Los consumidores no adquieren ropa, comidas o mobiliario para expresar quienes son, sino que construyen su identidad a través de los objetos de consumo, que adquieren un carácter simbólico (Baudrillard, 1997). La customización del coche, de los ordenadores o de la carcasa del móvil sirve para construir la identidad del consumidor. En este desarrollo social, el individuo se independiza cada vez más de las categorías sociales de origen y de pertenencia.

El quinto y último rasgo encontrado hace referencia a un nuevo tipo de consumidor más responsable. Si la modernidad clásica planteó la creencia en la ciencia y el desarrollo del consumo de masas, la modernidad tardía cuestiona el progreso e introduce el debate sobre el consumo responsable, la degradación del medio ambiente y el derroche. Inglehart y Welzel (2006) ponen de manifiesto que los valores y las creencias básicas de los públicos de las sociedades avanzadas difieren mucho de los de las sociedades menos desarrolladas, y estos valores están cambiando en una dirección previsible a medida que avanza el desarrollo económico. Afirman estos autores que, en términos de consumo, las sociedades postindustriales han conseguido tales niveles de bienestar que facilitan a casi todo el mundo comida, cobijo, ropa, vivienda, educación y servicios sanitarios. Este nivel de seguridad permite a las personas centrarse en metas que van más allá de la supervivencia inmediata. Por este motivo, con la 
maximización del nivel de vida en términos materiales, se persigue la maximización del bienestar a través del cambio en los estilos de vida. Y la calidad de la experiencia reemplaza a la cantidad de mercancías como principal criterio para vivir bien (Florida, 2002).

Los valores de autoexpresión se reflejan, en los últimos años, en nuevas teorías sobre el consumo ${ }^{4}$. En el contexto de nuevos movimientos como el consumerismo (Ramírez et al., 2002) y alternativas a una sociedad menos consumista (Klein, 2001), Alonso (2005: 103) nos dibuja a un nuevo consumidor que no sigue tanto las pautas de adhesión a grandes grupos como a círculos fuertemente particularistas. Discursos como la salud, la solidaridad, las relaciones sociales o la familia se mezclan con la compra misma, para organizar un escenario de opulencia controlada, responsabilidad y control, contra lo que pueden ser los efectos no queridos de un hedonismo mal entendido. Entre los grupos donde parece surgir, se encuentran las nuevas elites ascendentes. Un nuevo consumidor, cuya preocupación por los valores postmateriales, paradójicamente, le servirá para marcar nuevas diferencias respecto a otros grupos sociales.

En resumen, tras la revisión bibliográfica, el objetivo general de comparar las pautas de consumo en Andalucía y Cataluña (1995-2006) puede desglosarse en cuatro objetivos específicos:

1. Describir y comparar el acceso a los objetos de consumo en Andalucía y Cataluña y su evolución en el periodo 1995-2006. Conocer si, como afirma la teoría de la modernización, se produce una convergencia en el desarrollo. Si la sociedad andaluza se aproxima o se distancia de la sociedad catalana.

2. Analizar la igualdad social. Si, conforme se produce el desarrollo, las diferencias sociales respecto a la posesión de bienes de consumo se reducen.

3. Comprobar la tesis de la individuación. Si las categorías sociales explican cada vez menos la posesión de objetos de consumo.

4. Buscar patrones del nuevo consumidor. Si es posible encontrar un nuevo consumidor responsable, informado, menos consumista. En caso afirmativo, delimitar dónde se concentra esta nueva tendencia.

Se plantean nueve hipótesis para contrastar empíricamente:

- Hipótesis 1: Andalucía está más cerca de Cataluña en el año 2006 que en el año 1995.

- Hipótesis 2: La posesión de objetos de consumo es mayor en Cataluña que en Andalucía.

- Hipótesis 3: Hay una mayor posesión de objetos de consumo en 2006 que en 1995.

4. Hay que destacar la teoría de la modernización ecológica. Desarrollada por Joseph Huber, sostiene que, para minimizar o reducir el daño producido a los recursos naturales, son precisas reformas urgentes dentro de la sociedad moderna. 
- Hipótesis 4: Hay mayor igualdad social respecto al consumo en 2006 que en 1995.

- Hipótesis 5: Hay mayor igualdad social en Cataluña que en Andalucía.

- Hipótesis 6: Las categorías sociales explican menos en 2006 que en 1995.

- Hipótesis 7: Las categorías sociales explican menos en Cataluña que en Andalucía.

- Hipótesis 8: Es posible encontrar un nuevo consumidor que, teniendo posibilidades económicas y educativas, posea menos objetos de consumo. El nuevo consumidor será localizado en los grupos con mejor posición social.

- Hipótesis 9: El nuevo consumidor será localizado en mayor medida en Cataluña que en Andalucía.

\section{Metodología}

Para comprobar los objetivos y las hipótesis formuladas, se utiliza una base particular de datos de ASEP. Esta base de datos está formada a partir de la acumulación de las preguntas comunes en cada uno de los doce sondeos de opinión que lleva a cabo esta organización al año. Los años considerados son 1995 y 2006, tanto de Andalucía como de Cataluña ${ }^{5}$. El nivel de consumo se mide a través de dos indicadores: la extensión del consumo entre la población (porcentaje de población que consume los distintos bienes materiales) y el número de objetos de consumo que se poseen por término medio. Los objetos materiales que se utilizan para medir el consumo son los contenidos en la citada encuesta: vivienda propia, automóvil, teléfono fijo, lavadora, televisión en color, DVD, vídeo, teléfono móvil, equipo de sonido, ordenador, lavavajillas, Internet y conexión de ADSL. Para la elaboración del indicador de consumo medio, se han eliminado el televisión en color y la lavadora, que, por su universalización, no aparecen en la encuesta de 2006. También se ha eliminado el vídeo, puesto que los datos confirman que éste tiende a desaparecer de los hogares $^{6}$. Las variables utilizadas son: el nivel de ingresos del hogar, el nivel educativo del entrevistado, su estatus ocupacional ${ }^{7}$, el hábitat de residencia, la

5. La muestra para Andalucía es de 2.385 encuestados en el año 1995 y de 2.297 en el año 2006. En el caso de Cataluña, la muestra es de 2.094 en el año 1995 y de 2.123 en el año 2006.

6. Metodológicamente, no sería comparable la situación de 1995 con la de 2006, puesto que en este último año hay más objetos de consumo. Sin embargo, entendemos que, en cada periodo histórico, los objetos de consumo que distinguen varían, por lo que sería un sinsentido tener en cuenta en el año 2006 la posesión de los objetos que en 1995 eran más destacados y olvidar aquellas nuevas tecnologías que han surgido. Junto con ello, creemos que es aceptable este modo de comparación, puesto que lo que se mide no es estrictamente el aumento de objetos de consumo en 1995-2006, sino si en ambos periodos las categorías sociales explican el consumo, si hay una mayor igualdad social y si las diferencias entre Andalucía y Cataluña son mayores o menores.

7. Según la clasificación de ASEP. 
edad, el índice de posición social ${ }^{8}$ y la escala de valores materialistas y postmaterialistas. Para comparar los resultados de Andalucía y Cataluña, así como su evolución, se ha elaborado una tasa que asigna el valor 100 al grupo con menor número medio de objetos de consumo y, a partir de éste, se compara el resto (cuadros 3 a 10).

\section{Resultados}

\subsection{Diferencias y evolución en el acceso a los bienes de consumo entre la población general de Andalucia y Cataluña (1995-2006)}

La investigación de Tezanos (1984) permite comparar Andalucía y Cataluña. En el período 1980-1981, Cataluña era la segunda región con mayor nivel de consumo, y Andalucía, la tercera, a la cola, con menor nivel de consumo. Si en Cataluña el porcentaje de población con automóvil alcanzaba el 61,1\%, en Andalucía éste resultaba del $42,7 \%$; la televisión en color estaba extendida entre el $35,8 \%$ y el $24 \%$ de catalanes y andaluces, respectivamente; la lavadora automática, entre el 76,7\% y el 56,4\%; el lavavajillas alcanzaba al 7,6\% de los hogares catalanes y al 3,7\% de los andaluces. En Cataluña, solamente el $1 \%$ de la población en 1981 carecía en su hogar de agua corriente y de servicios de higiene; no existían hogares sin electricidad y los que carecían de teléfono alcanzaban el 34\%. En Andalucía, los hogares sin agua corriente eran el $6 \%$; sin servicios de higiene, el 10\%; sin electricidad, un 2\%, y sin teléfono, hasta el 69\% (Tezanos, 1984). En la actualidad, se ha alcanzado un nivel de desarrollo que impide emplear estos indicadores para comparar Andalucía y Cataluña. Se siguen utilizando para medir el desarrollo y la pobreza en muchos países (IDH, Naciones Unidas), mientras que se buscan otros que sirvan para comparar el desarrollo alcanzado en sociedades con bienestar material.

El cuadro 1 refleja que, con el paso de los años, se produce una extensión de los objetos de consumo entre amplias capas de población. Es lo que ha ocurrido desde 1995 hasta 2006. Los objetos de consumo que se implantaron en los años sesenta, como la lavadora o la televisión en color, han alcanzado una extensión prácticamente universal. Tras ellos se encuentra la vivienda en propiedad. El porcentaje de población que accede a una vivienda en propiedad ha pasado del $78,7 \%$ al $84,7 \%$ en tan solo una década. El porcentaje de población que posee un vehículo, marcado símbolo de estatus, tampoco deja de cre-

8. Es un índice sintético que sigue el sistema diseñado por Galtung. El índice de posición social se obtiene de la combinación de diferentes variables: sexo, edad, ocupación del entrevistado, educación del entrevistado, provincia de residencia, hábitat de residencia y nivel de ingresos en el hogar. El índice varía de 0 a 8 puntos tras combinar dichas variables: asignan un punto a las características siguientes: a los varones; a los de 30 a 64 años; trabajadores no manuales, de nivel de ocupación alto y medio, a los del sector de la industria y de los servicios del sector público, del sector privado; a los de nivel educativo alto, y a los residentes en un hábitat urbano y metropolitano y en determinadas provincias. 
Cuadro 1. Porcentaje de población que accede a los objetos de consumo y media de objetos de consumo por hogar en Andalucía, Cataluña y España (1995-2006)

\begin{tabular}{|c|c|c|c|c|c|c|}
\hline \multirow[b]{2}{*}{ Bienes de consumo } & \multicolumn{2}{|c|}{ Andalucía } & \multicolumn{2}{|c|}{ Cataluña } & \multicolumn{2}{|c|}{ España } \\
\hline & 1995 & 2006 & 1995 & 2006 & 1995 & 2006 \\
\hline TV en color & 97,6 & No & 98,3 & No & 97,5 & No \\
\hline Lavadora & 95,0 & No & 96,8 & No & 95,8 & No \\
\hline Vivienda en propiedad & 78 & 80,1 & 73,5 & 87,3 & 78,7 & 84,7 \\
\hline Móvil & No & 76,4 & No & 86,6 & No & 78,2 \\
\hline Teléfono fijo & 67,1 & 60,1 & 87,1 & 87,1 & 81,4 & 76,0 \\
\hline Automóvil & 61,2 & 70,5 & 69,6 & 79,9 & 67,7 & 73,8 \\
\hline DVD & No & 65,2 & No & 83,4 & No & 67,3 \\
\hline Equipo de sonido & 36,2 & 54,2 & 49,2 & 82,5 & 42,7 & 61,3 \\
\hline Vídeo & 55,3 & 53,5 & 66,3 & 64,1 & 61,5 & 56,9 \\
\hline Ordenador & 11,2 & 38,6 & 17,0 & 52,1 & 14,6 & 44,0 \\
\hline Lavavajillas & 10,3 & 29,6 & 12,8 & 27,1 & 15,0 & 31,7 \\
\hline Internet & No & 15,8 & No & 30,0 & No & 22,3 \\
\hline ADSL & No & 11,4 & No & 27,1 & No & 17,4 \\
\hline Media de consumo & 2,64 & 5,02 & 3,09 & 6,43 & 3,00 & 5,57 \\
\hline Porcentaje sobre total objetos & 44 & 50,2 & 51,5 & 64,3 & 50,0 & 55,7 \\
\hline
\end{tabular}

Fuente: elaboración propia a partir del sondeo de opinión pública de ASEP (1995-2006).

cer, pese a los discursos medioambientales que recomiendan un uso limitado de él o los problemas de tráfico que genera la utilización del transporte privado en las ciudades. Si en el año 1995 el 67,7\% de los españoles tenía un vehículo, en el 2006 esta cifra alcanza el 73,8\%.

El cuadro 1 también da cuenta de que nos hallamos inmersos en sociedades tecnológicas. Tras la superación de la fase de implantación del automóvil y de los objetos que agilizaban las tareas domésticas en la España de los años sesenta, setenta y ochenta, desde los años noventa se implantan aceleradamente nuevos objetos tecnológicos en la sociedad del conocimiento. Y una característica de tales objetos de consumo, en una sociedad caracterizada por el cambio, es que los avances tecnológicos sustituyen a unos objetos de consumo por otros, y estos últimos dejan de ser necesarios y quedan relegados a un segundo plano: desfasados, pasados de moda y superfluos ${ }^{9}$. Es el caso del vídeo, que, sin llegar a extenderse universalmente en la década de 1990 (61,5\%), hoy es

9. Daniel Bell anticipaba, en El advenimiento de la sociedad post-industrial, que una de las dimensiones de esta nueva sociedad era la planificación de la tecnología. "Una sociedad moderna, para evitar el estancamiento o la "madurez" (con toda la imprecisión de esta palabra), ha tenido que explorar nuevas fronteras tecnológicas con el fin de mantener la productividad y los niveles de vida elevados» (1983:44 [1973]). 
eliminado del hogar $(56,9 \%)$ y sustituido progresivamente por el DVD, que se impone entre el 67,3\% de la población. Igual ocurre en una sociedad «móvil» con la telefonía que ya no necesita cables ni un espacio permanente. El teléfono fijo comienza a ser eliminado del hogar (en el periodo estudiado, pasa del $81,4 \%$ al $76 \%$ ), mientras que el móvil aparece en escena y, en un periodo fugaz de tiempo, se hace imprescindible para el $78,2 \%$ de la población.

Pese a las novedades surgidas en los últimos años, hay ciertas pautas de consumo que permanecen. Y una de ellas es que constantemente surgen nuevos objetos que pasan de ser elitistas a consumirse de modo generalizado. En el año 1995, solo un 14,6\% de los españoles se declaraba poseedor de un ordenador. En el año 2006, la cifra alcanza el 44\%, mientras que las políticas sociales persiguen aumentar esta cantidad como indicador de modernización y de colectivos tecnológicamente avanzados. Pero aún son pocos los hogares conectados al ciberespacio. La conexión a Internet en el hogar está limitada al 22,3\% de las viviendas y tan solo un 17,4\% disfruta de ADSL.

Centrándonos en la comparación entre Cataluña y Andalucía, tal y como se esperaba, en Cataluña hay una mayor extensión de los objetos de consumo entre la población. En todos los objetos de consumo, Cataluña sobresale por encima del total de España y Andalucía. Andalucía muestra una extensión de los bienes de consumo más limitada. Las diferencias se acrecientan en los objetos tecnológicos de consumo, que sitúan a Cataluña próxima a las sociedades tecnológicas y muestran "La brecha digital en Andalucía» (Bericat y López Menchón, 2006).

Los datos nos permiten resumir que, en Cataluña, aproximadamente ocho de cada diez ciudadanos cuentan con la mayor parte de los objetos tecnológicos existentes en el mercado, y que más de la mitad $(52,1 \%)$ tiene ordenador. En Andalucía, estos resultados se reducen a seis de cada diez, mientras que el ordenador es propiedad del 38,6\% de sus habitantes. Encontramos también diferencias en la extensión del teléfono móvil en Andalucía (76,4\%) y Cataluña (86,6\%); en el DVD $(65,2 \%$ y $83,4 \%$, respectivamente) o en el equipo de sonido (54,2\% y 82,5\%). Otro dato. En Andalucía el teléfono fijo es eliminado progresivamente del $67,1 \%$ hasta el $60,1 \%$ de la población. En Cataluña se mantiene en el $87,1 \%$ de los hogares. Hogares muchos de ellos que, a falta de datos, han implantado o tienen previsto implantar conexión a Internet y en muchos casos necesitan aún mantener el teléfono fijo. En Cataluña, el porcentaje de hogares que tiene conexión a Internet (30\%) duplica al de Andalucía $(15,8 \%)$ y las diferencias son aún mayores si la conexión es a través de ADSL ( $27,1 \%$ y $11,4 \%$, respectivamente). La única excepción la constituye un electrodoméstico, el lavavajillas, que está algo más extendido en la comunidad autónoma andaluza. Se podría pensar, sin contrastación empírica, que algunas variables estructurales pueden ser explicativas de este fenómeno. Andalucía cuenta, comparativamente, con mayor número medio de hijos por mujer, un mayor número de hogares de familia extensa y menor número de hogares unipersonales (Bericat y Martín-Lagos, 2006).

Los catalanes cuentan, por término medio, con más objetos en el hogar $(6,43)$, por encima de la media española $(5,57)$ y andaluza $(5,02)$. Resultados 
que nos llevan a afirmar que, si el nivel de desarrollo se mide a través de la posesión de objetos de consumo y de su universalización entre amplias capas de población, Cataluña sigue estando más próxima a este desarrollo que Andalucía y que la media española. En 1995, de los 6 objetos comparados, los hogares andaluces contaban por término medio con un $44 \%$ y los catalanes, con un $51,5 \%$. En el año 2006, de los 10 objetos comparados, Andalucía cuenta con el 50,2\%. Cataluña ha seguido creciendo a un ritmo más rápido que Andalucía y, en el año 2006, sus hogares cuentan con el 64,3\% de los objetos. Las diferencias entre ambas son mayores $(14,1 \%)$ que hace una década (7,5\%), de modo que, en el año 2006, se puede afirmar que Andalucía está más lejos de Cataluña que en 1995.

\subsection{Las categorías sociales explicativas del acceso a los objetos de consumo}

\subsubsection{Resultados de la comparación de medias}

La teoría de la modernización sostiene que, al aumentar el desarrollo, las categorías sociales explican en menor medida el tipo de consumo. Según nuestra hipótesis de partida, las categorías sociales explicarán menos en Cataluña que en Andalucía y menos en 2006 que en 1995. Para comprobar la hipótesis citada, se realiza una comparación de medias entre el número de objetos de consumo y las variables hábitat, nivel de ingresos del hogar, nivel educativo, estatus ocupacional, escala de posición social, escala de materialismo y postmaterialismo y edad del entrevistado.

La comparación de medias se realiza separadamente entre cada comunidad autónoma y en los años 1995-2006 (cuadro 2). En Andalucía, las diferencias de medias resultan significativas en todas las variables analizadas. Las categorías sociales siguen explicando la cantidad de objetos de consumo que se poseen. En Cataluña, el hábitat es la única variable que no explica el comportamiento ante el consumo. En 1995 y en Andalucía, las variables que más explicaban el comportamiento eran el nivel de ingresos (28,7\%), el nivel educativo $(21,8 \%)$ y la posición social $(10,8 \%)$. El resto mostraba una asociación más débil y explicaba muy poco el consumo. En Cataluña, ocurría algo similar, aunque explicaban algo menos que en Andalucía, y a las tres variables mencionadas había que sumarles la edad (10,7\%).

En el año 2006, contrariamente a lo que se pudiera pensar, las categorías sociales siguen explicando la cantidad de objetos de consumo que se poseen. En Andalucía, el nivel de ingresos y el nivel educativo explican menos que antes y, en su lugar, la edad tiene cada vez más importancia como variable explicativa. En Cataluña, el nivel de ingresos también explica cada vez menos, aunque, a diferencia de Andalucía, es la educación la que ahora explica más el comportamiento $(34,1 \%)$. La edad también adquiere cada vez más protagonismo $(26,9 \%)$. En resumen, se puede afirmar que las categorías sociales siguen explicando el comportamiento ante el consumo, contrariamente a lo que postula la teoría de la modernización. Además, en el año 2006, las categorías sociales explican más en Cataluña que en Andalucía. 
Cuadro 2. Resultados de la comparación de medias entre la media de objetos de consumo y distintas variables. Tabla ANOVA, eta y eta cuadrado

\begin{tabular}{|c|c|c|c|c|c|c|c|c|c|}
\hline \multicolumn{10}{|c|}{ Andalucía } \\
\hline \multicolumn{5}{|c|}{1995} & \multicolumn{5}{|c|}{2006} \\
\hline Variables & $\mathrm{F}$ & Sig. & Eta & $\begin{array}{l}\text { Eta } \\
\text { cuad. }\end{array}$ & Variables & $\mathrm{F}$ & Sig. & Eta & $\begin{array}{l}\text { Eta } \\
\text { cuad. }\end{array}$ \\
\hline Ingresos & 95,036 & 0,000 & 0,536 & 0,287 & Ingresos & 66,391 & 0,000 & 0,519 & 0,269 \\
\hline Nivel educativo & 82,610 & 0,000 & 0,467 & 0,218 & Nivel educativo & 67,032 & 0,000 & 0,436 & 0,190 \\
\hline Posición social & 36,081 & 0,000 & 0,329 & 0,108 & Edad & 128,441 & 0,000 & 0,379 & 0,144 \\
\hline Estatus ocupacional & 30,537 & 0,000 & 0,282 & 0,079 & Posición social & 41,172 & 0,000 & 0,355 & 0,126 \\
\hline Edad & 65,901 & 0,000 & 0,277 & 0,077 & $\begin{array}{l}\text { Materialismo } \\
\text { y postmaterialismo }\end{array}$ & 20,499 & 0,000 & 0,207 & 0,043 \\
\hline $\begin{array}{l}\text { Materialismo } \\
\text { y postmaterialismo }\end{array}$ & 20,971 & 0,000 & 0,205 & 0,042 & Estatus ocupacional & 17,387 & 0,000 & 0,176 & 0,031 \\
\hline Hábitat & 47,48 & 0,000 & 0,196 & 0,038 & Hábitat & 10,549 & 0,000 & 0,095 & 0,009 \\
\hline \multicolumn{10}{|c|}{ Cataluña } \\
\hline \multicolumn{5}{|c|}{1995} & \multicolumn{5}{|c|}{2006} \\
\hline Variables & $\mathrm{F}$ & Sig. & Eta & $\begin{array}{l}\text { Eta } \\
\text { cuad. }\end{array}$ & Variables & $\mathrm{F}$ & Sig. & Eta & $\begin{array}{l}\text { Eta } \\
\text { cuad. }\end{array}$ \\
\hline Ingresos & 69,486 & 0,000 & 0,532 & 0,283 & Nivel educativo & 136,652 & 0,000 & 0,584 & 0,341 \\
\hline Nivel educativo & 65,898 & 0,000 & 0,449 & 0,202 & Ingresos & 22,852 & 0,000 & 0,530 & 0,281 \\
\hline Edad & 83,775 & 0,000 & 0,328 & 0,107 & Edad & 260,548 & 0,000 & 0,519 & 0,269 \\
\hline Posición social & 29,330 & 0,000 & 0,318 & 0,101 & Posición social & 46,101 & 0,000 & 0,385 & 0,149 \\
\hline Estatus ocupacional & 26,070 & 0,000 & 0,252 & 0,063 & $\begin{array}{l}\text { Materialismo } \\
\text { y postmaterialismo }\end{array}$ & 35,843 & 0,000 & 0,279 & 0,078 \\
\hline $\begin{array}{l}\text { Materialismo } \\
\text { y postmaterialismo }\end{array}$ & 8,250 & 0,000 & 0,139 & 0,019 & Estatus ocupacional & 20,780 & 0,000 & 0,189 & 0,036 \\
\hline Hábitat & 1,540 & 0,215 & 0,038 & 0,001 & Hábitat & 1,676 & 0,187 & 0,40 & 0,002 \\
\hline
\end{tabular}

Fuente: elaboración propia a partir de la base de datos de ASEP (1995-2006).

3.2.2. Variables más explicativas: nivel de ingresos ${ }^{10}$, nivel educativo y edad

El nivel de ingresos ha sido una de las variables que ha explicado tradicionalmente el consumo de bienes materiales. En el año 1995, en Andalucía, los que

10. Hay que tener en cuenta algunas cuestiones metodológicas a la hora de interpretar la variable ingresos. Los extremos suelen tener pocos casos y no siempre resultan representativos. En Andalucía, en 1995, en el grupo de ingresos >3.000 euros hay 6 casos; en 2006, en Andalucía, en el grupo de $<300$ euros, 8 casos; en el de $>3.000,10$ casos. En Cataluña, la muestra de 1995 no reviste problemas. La de 2006 tiene $\mathrm{N}=477$ y un elevado número de No respuesta $=1.646$. En este mismo año, los que ganan $<300$ euros son solo 6 casos; los que ganan más de 3.000 euros son 7 casos. 
menos ingresos tenían (menos de 300 euros) disponían de más objetos de consumo que en Cataluña. E igual ocurría con los que ganaban más de 1.200 euros. En el año 2006, en Cataluña, las diferencias entre grupos son menores. En Andalucía han aumentado y han seguido similar comportamiento al del conjunto de España. El mapa de 2006 se hace más difícil de comprender. Tener más o menos ingresos ya no implica tener más o menos objetos de consumo, lo que nos remite directamente al concepto de estilo de vida. Un hecho que llama la atención es que, en Cataluña, el grupo cuyos ingresos superan los 3.000 euros posee menos objetos de consumo que el anterior, algo que no ocurre en Andalucía ni en el conjunto de España. En Andalucía, tener dinero equivale a poseer objetos de consumo. Incluso si se tiene poco, se aspira a poseerlos. En Cataluña, tener mucho dinero no implica disponer de todos los objetos materiales. Se puede buscar el acceso a otros bienes inmateriales (viajes, emociones, etc.), lo que nos remite directamente a los valores postmaterialistas ${ }^{11}$.

Junto con el nivel de ingresos, la educación ha desempeñado un importante papel en el modo de utilizar los bienes de consumo (Bourdieu, 1979). $\mathrm{Al}$ aumentar el nivel de estudios del entrevistado, también lo hace el número de objetos de consumo que se poseen (cuadro 3). Comparando ambas comunidades autónomas según la base 100, en el año 1995, en Cataluña, las diferencias entre el nivel de estudios más bajo y el más alto eran menores que en Andalucía. Pero las diferencias han ido aumentando, de manera que el nivel educativo en Cataluña marca más diferencias hoy que hace una década. Probablemente porque las tecnologías muestran más diferencias según el nivel educativo que los objetos de consumo de los años noventa.

En último lugar, se encuentra la edad. Desde la aparición de los Teddy Boys en el Reino Unido en la década de 1950, asociados con el rock and roll y un modo de vestir característico (Yates, 2002), comienza a hablarse de la cultura juvenil. Es especialmente a partir de los años ochenta y noventa cuando la edad se utiliza en las investigaciones de mercado como variable explicativa del tipo de consumo (Bocock, 1995). Los resultados muestran que, en nuestra sociedad, ser joven equivale a ser un gran consumidor, especialmente de productos tecnológicos. Ya en 1982, José Castillo hacía referencia a esta situación al hablar de los «hijos de la sociedad de consumo española» ${ }^{12}$. Además, los años de vida explican cada vez más los patrones de consumo, de manera que las diferencias

11. Inglehart mencionaba, en Modernización y posmodernización, el cambio en los valores sociales. Desde la prioridad al auge económico hasta la maximización de la autoexpresión y la valoración de la calidad de la experiencia (1999:75-76). No obstante, hay que tener precaución con los resultados, puesto que en algunas casillas (las sombreadas) la muestra es inferior a 20 casos.

12. Nuestros hijos y nietos se zambullen de buenas a primeras, y como la cosa más natural del mundo, en la esfera de los cacharros electrónicos [...] no hay comparación posible: ninguno de los seres de carne y hueso con los que a diario convive el niño pueden competir con las máquinas, que se saben todos los cuentos imaginables, que cantan canciones modernas [...] Otro asunto es que estos precoces consumidores acaben por aburrirse rápida e irremediablemente» (Castillo, 1982: 51). 
Cuadro 3. Número medio de objetos de consumo en Andalucía, Cataluña y España, según el nivel de ingresos del hogar, el nivel educativo y la edad del entrevistado (1995-2006)

\begin{tabular}{|c|c|c|c|c|c|c|c|c|c|c|c|c|}
\hline \multicolumn{13}{|c|}{ Variables más explicativas } \\
\hline \multirow{3}{*}{$\begin{array}{l}\text { Ingresos } \\
\text { en el hogar } \\
<300\end{array}$} & \multicolumn{6}{|c|}{1995} & \multicolumn{6}{|c|}{2006} \\
\hline & \multicolumn{2}{|c|}{ Andalucía } & \multicolumn{2}{|c|}{ Cataluña } & \multicolumn{2}{|c|}{ España } & \multicolumn{2}{|c|}{ Andalucía } & \multicolumn{2}{|c|}{ Cataluña } & \multicolumn{2}{|l|}{ España } \\
\hline & 1,70 & 100 & 1,53 & 100 & 1,80 & 100 & 2,71 & 105,0 & 6,00 & 188,1 & 3,33 & 128,6 \\
\hline $301-450$ & 1,87 & 110 & 1,94 & 126,8 & 2,00 & 111,1 & 2,58 & 100 & 3,19 & 100 & 2,59 & 100 \\
\hline $451-600$ & 2,46 & 144,7 & 2,57 & 168,0 & 2,57 & 142,8 & 3,00 & 116,3 & 4,23 & 132,6 & 2,88 & 111,2 \\
\hline $601-900$ & 2,92 & 171,8 & 3,03 & 198,0 & 3,07 & 170,6 & 4,50 & 174,4 & 5,20 & 163,0 & 4,37 & 168,7 \\
\hline $901-1200$ & 3,50 & 205,9 & 3,56 & 232,7 & 3,56 & 197,8 & 5,76 & 223,3 & 5,33 & 167,1 & 5,52 & 213,1 \\
\hline $1201-1650$ & 4,12 & 242,3 & 4,03 & 263,4 & 4,05 & 225,0 & 5,77 & 223,6 & 6,73 & 211,0 & 6,43 & 248,3 \\
\hline $1651-2100$ & 4,66 & 274,1 & 4,25 & 277,8 & 4,38 & 243,3 & 6,47 & 250,8 & 7,83 & 245,4 & 7,13 & 275,3 \\
\hline $2101-3000$ & 4,67 & 274,7 & 4,58 & 299,3 & 4,60 & 255,6 & 7,46 & 289,1 & 8,29 & 259,9 & 7,86 & 303,5 \\
\hline$>3000$ & 4,62 & 271,8 & 4,50 & 294,1 & 4,86 & 270,0 & 8,30 & 321,7 & 6,25 & 195,2 & 8,28 & 319,7 \\
\hline Total & 2,52 & 100 & 2,98 & 118,2 & 2,90 & 115,1 & 5,05 & 100 & 5,75 & 113,9 & 5,38 & 106,5 \\
\hline $\begin{array}{l}\text { Eta cuadrado } \\
\text { y N }\end{array}$ & 0,287 & 1.895 & 0,283 & 1.416 & 0,295 & 10.020 & 0,269 & 1.452 & 0,281 & 477 & 0,353 & 7.985 \\
\hline \multicolumn{13}{|l|}{$\begin{array}{l}\text { Nivel } \\
\text { educativo }\end{array}$} \\
\hline No sabe leer & 1,65 & 100,0 & 1,80 & 100 & 1,75 & 100 & 2,53 & 100 & 3,00 & 100 & 2,64 & 100,0 \\
\hline Sabe leer & 2,08 & 126,1 & 2,31 & 128,1 & 2,30 & 131,2 & 3,27 & 129,2 & 3,41 & 113,7 & 2,88 & 109,2 \\
\hline $\begin{array}{l}\text { Primarios } \\
\text { completos }\end{array}$ & 2,60 & 157,9 & 2,72 & 150,9 & 2,79 & 159,6 & 4,63 & 183,0 & 4,40 & 146,7 & 4,23 & 160,4 \\
\hline EGB o EPO & 3,19 & 193,5 & 3,46 & 192,3 & 3,29 & 188,0 & 5,42 & 214,2 & 6,23 & 207,7 & 5,59 & 211,6 \\
\hline FP o módulos & 3,15 & 191,0 & 3,69 & 205,1 & 3,48 & 199,1 & 5,82 & 230,0 & 7,17 & 239,0 & 6,47 & 244,9 \\
\hline BUP o ESO & 3,14 & 190,6 & 3,18 & 176,9 & 3,26 & 186,3 & 5,45 & 215,4 & 7,47 & 249,0 & 6,51 & 246,8 \\
\hline $\begin{array}{l}\text { COU } \\
\text { o bachiller }\end{array}$ & 3,60 & 218,5 & 3,85 & 214,1 & 3,75 & 214,4 & 6,76 & 267,2 & 8,01 & 267,0 & 7,17 & 271,5 \\
\hline Grado medio & 3,77 & 228,3 & 3,78 & 209,9 & 3,87 & 221,3 & 6,50 & 256,9 & 7,52 & 250,7 & 6,92 & 262,2 \\
\hline Universitarios & 3,94 & 238,6 & 4,19 & 232,9 & 4,20 & 239,7 & 6,74 & 266,4 & 8,05 & 268,3 & 7,39 & 279,9 \\
\hline Total & 2,64 & 100 & 3,09 & 117,0 & 3,00 & 113,6 & 5,02 & 100 & 6,43 & 128,1 & 5,57 & 111,0 \\
\hline $\begin{array}{l}\text { Eta cuadrado } \\
\text { y N }\end{array}$ & 0,218 & 2.381 & 0,202 & 2.091 & 0,198 & 13.312 & 0,190 & 2.289 & 0,341 & 2.117 & 0,289 & 13.177 \\
\hline
\end{tabular}


Cuadro 3. Número medio de objetos de consumo en Andalucía, Cataluña y España, según el nivel de ingresos del hogar, el nivel educativo y la edad del entrevistado (1995-2006) (continuación)

\begin{tabular}{|c|c|c|c|c|c|c|c|c|c|c|c|c|}
\hline \multicolumn{13}{|c|}{ Variables más explicativas } \\
\hline \multirow{3}{*}{$\begin{array}{l}\text { Edad } \\
\text { Jóvenes }\end{array}$} & \multicolumn{6}{|c|}{1995} & \multicolumn{6}{|c|}{2006} \\
\hline & \multicolumn{2}{|c|}{ Andalucía } & \multicolumn{2}{|c|}{ Cataluña } & \multicolumn{2}{|c|}{ España } & \multicolumn{2}{|c|}{ Andalucía } & \multicolumn{2}{|c|}{ Cataluña } & \multicolumn{2}{|c|}{ España } \\
\hline & 2,92 & 160,4 & 3,47 & 154,1 & 3,29 & 148,7 & 5,62 & 187,3 & 7,32 & 173,1 & 6,39 & 190,6 \\
\hline $\begin{array}{l}\text { Adultos } \\
\text { jóvenes }\end{array}$ & 2,81 & 154,8 & 3,44 & 152,7 & 3,28 & 148,3 & $\underline{5,77}$ & 192,2 & 7,26 & 171,6 & 6,44 & 192,1 \\
\hline $\begin{array}{l}\text { Adultos } \\
\text { mayores }\end{array}$ & 2,64 & 145,2 & 2,96 & 131,6 & 3,00 & 135,7 & 5,03 & 167,5 & 6,53 & 154,5 & 5,69 & 169,9 \\
\hline $3^{\mathrm{a}}$ y $4^{\mathrm{a}}$ edad & 1,82 & 100,0 & 2,25 & 100,0 & 2,21 & 100,0 & 3,00 & 100,0 & 4,23 & 100,0 & 3,35 & 100,0 \\
\hline Total & 2,64 & 100,0 & 3,09 & 117,0 & 3,00 & 113,6 & 5,02 & 100,0 & 6,43 & 128,1 & 5,57 & 111,0 \\
\hline $\begin{array}{l}\text { Eta cuadrado } \\
\text { y N }\end{array}$ & 0,077 & 2.385 & 0,107 & 2.094 & 0,081 & 13.356 & 0,144 & 2.297 & 0,269 & 2.123 & 0,211 & 3.245 \\
\hline
\end{tabular}

Fuente: elaboración propia a partir del sondeo de opinión pública de ASEP (1995-2006).

1. La comparación de medias según la variable nivel de ingresos resulta significativa en ambas comunidades autónomas y en España en los años 1995 y 2006.

2. Solamente contamos con un 22,5\% de respuesta en Cataluña en 2006.

entre los jóvenes y la tercera y la cuarta edades son cada vez mayores. Las diferencias según edad eran y siguen siendo mayores en Andalucía que en Cataluña. En Andalucía sorprende el hecho de que ya no son los más jóvenes los mayores consumistas, sino que han cedido su puesto a los jóvenes adultos. Esta pauta hace pensar que la generación de jóvenes socializados hace una década en las pautas de consumo ha continuado consumiendo con diez años más.

\subsubsection{Variables menos explicativas del consumo: ocupación, hábitat y valores del entrevistado}

En el cuadro 4 se ofrecen los resultados de tres variables que explican poco las diferencias en el consumo: la ocupación, el hábitat y los valores del entrevistado. Las dos primeras son cada vez menos explicativas; la tercera, aunque aún explica poco, muestra una tendencia ascendente. A finales de los años sesenta, el estudio de Goldthorpe ponía de relieve la influencia que ejercía la ocupación en el tipo de consumo (Goldthorpe et al., 1968), pero, desde la década de los años ochenta, existe la percepción de que la ocupación cada vez explica menos el tipo de consumo (Bocock, 1995: 46-47).

Los resultados de nuestra investigación (cuadro 2) son acordes con la teoría: la ocupación aún explica el tipo de consumo, aunque cada vez menos. Al aumentar el estatus ocupacional, también lo hace el número medio de objetos de consumo que se poseen. Pero en el año 2006 los grupos están más cercanos, de modo que la ocupación cada vez predice menos el consumo. En ambas comunidades se tiende a la igualación, aunque en Andalucía la ocupación ha marcado y sigue marcando más distancias en el consumo. 
Cuadro 4. Número medio de objetos de consumo en Andalucía, Cataluña y España, según el estatus ocupacional, el hábitat y los valores del entrevistado (1995-2006)

\begin{tabular}{|c|c|c|c|c|c|c|c|c|c|c|c|c|}
\hline \multicolumn{13}{|c|}{ Variables menos explicativas } \\
\hline \multirow{3}{*}{$\begin{array}{l}\text { Estatus } \\
\text { ocupacional } \\
\text { Bajo }\end{array}$} & \multicolumn{6}{|c|}{1995} & \multicolumn{6}{|c|}{2006} \\
\hline & \multicolumn{2}{|c|}{ Andalucía } & \multicolumn{2}{|c|}{ Cataluña } & \multicolumn{2}{|c|}{ España } & \multicolumn{2}{|c|}{ Andalucía } & \multicolumn{2}{|c|}{ Cataluña } & \multicolumn{2}{|l|}{ España } \\
\hline & 2,28 & 100,0 & 2,79 & 100,0 & 2,67 & 100,0 & 5,13 & 100,0 & 6,65 & 100,0 & 5,81 & 100,0 \\
\hline Medio & 3,08 & 135,4 & 3,52 & 126,2 & 3,36 & 126,1 & 5,71 & 111,4 & 7,34 & 110,3 & 6,49 & 111,8 \\
\hline Alto & 3,79 & 166,4 & 4,21 & 151,0 & 4,20 & 157,6 & 6,50 & 126,8 & 7,72 & 116,0 & 7,28 & 125,4 \\
\hline Total & 2,95 & 100,0 & 3,51 & 119,0 & 3,35 & 113,6 & 5,59 & 100,0 & 7,16 & 128,1 & 6,38 & 114,1 \\
\hline $\begin{array}{l}\text { Eta cuadrado } \\
\text { y N }\end{array}$ & 0,079 & 712 & 0,063 & 775 & 0,072 & 4.636 & 0,031 & 1.091 & 0,036 & 1.124 & 0,044 & 6.349 \\
\hline \multicolumn{13}{|l|}{ Hábitat* } \\
\hline Rural & 2,42 & 100 & 3,03 & 100 & 2,78 & 100 & 4,76 & 100 & 6,46 & 101,9 & 5,07 & 100 \\
\hline Semiurbano & 2,44 & 100,8 & 3,05 & 100,7 & 2,94 & 105,7 & 4,87 & 102,3 & 6,53 & 103 & 5,52 & 108,9 \\
\hline Urbano & 2,99 & 123,5 & 3,15 & 104 & 3,17 & 114 & 5,32 & 111,8 & 6,34 & 100 & 5,88 & 116 \\
\hline Total & 2,62 & 100 & 3,08 & 117,6 & 3,00 & 114,5 & 5,02 & 100 & 6,43 & 128,1 & 5,57 & 110,9 \\
\hline $\begin{array}{l}\text { Eta cuadrado } \\
\text { y N }\end{array}$ & 0,038 & 2.385 & 0,001 & 2.091 & 0,013 & 13.353 & 0,009 & 2.297 & 0,002 & 2.123 & 0,0151 & 13.242 \\
\hline \multicolumn{13}{|c|}{$\begin{array}{l}\text { Materialismo } \\
\text { y postmaterialismo }\end{array}$} \\
\hline 0. & 2,29 & 100,0 & 2,87 & 100,0 & 2,56 & 100,0 & 4,07 & 100,0 & 5,37 & 100,0 & 5,09 & 100,0 \\
\hline 1. & 2,40 & 104,8 & 2,92 & 101,8 & 2,79 & 109,1 & 4,54 & 111,6 & 5,30 & 98,6 & 5,06 & 99,5 \\
\hline 2. & 2,68 & 117,4 & 3,06 & 106,7 & 3,02 & 118,2 & 5,23 & 128,3 & 6,35 & 118,2 & 5,65 & 111,2 \\
\hline 3. & 2,97 & 129,9 & 3,13 & 109,1 & 3,18 & 124,3 & 5,49 & 134,8 & 6,85 & 127,5 & 5,80 & 114,0 \\
\hline 4. & 3,16 & 138,1 & 3,58 & 124,8 & 3,47 & 135,8 & 5,71 & 140,1 & 7,46 & 138,9 & 6,11 & 120,1 \\
\hline $\begin{array}{l}\text { 5. Postmateria- } \\
\text { listas }\end{array}$ & 3,32 & 145,4 & 3,61 & 125,8 & 3,54 & 138,2 & 6,77 & 166,2 & 7,08 & 131,7 & 5,92 & 116,3 \\
\hline Total & 2,64 & 100 & 3,09 & 117,0 & 3,00 & 113,6 & 5,02 & 100 & 6,43 & 128,1 & 5,57 & 111,0 \\
\hline $\begin{array}{l}\text { Eta cuadrado } \\
\text { y N }\end{array}$ & 0,042 & 2.385 & 0,019 & 2.094 & 0,033 & 13.356 & 0,043 & 2.297 & 0,078 & 2.123 & 0,0171 & 13.245 \\
\hline
\end{tabular}

Fuente: elaboración propia a partir de la base de datos de ASEP (1995-2006).

* El hábitat no es estadísticamente significativo en Cataluña en 1995 y en 2006 . Ver el cuadro 3. 
En segundo lugar, se comenta el hábitat ${ }^{13}$. No es una novedad afirmar que el hábitat ha sido una variable explicativa del desarrollo. Históricamente, la sociedad industrial trajo consigo el proceso de urbanización o emigración del campo a la ciudad. Las ciudades comenzaron a desarrollarse económicamente alrededor de las industrias, que, como nuevos motores de la economía, atraían el capital. La movilidad y la innovación crecieron alrededor de estos núcleos y las zonas rurales quedaron atrasadas y muchas veces faltas de infraestructuras (De Miguel, 1998). Sin embargo, la realidad actual refleja que, progresivamente, las zonas rurales se han ido urbanizando y se han acuñado nuevos términos para designar a las ciudades contemporáneas ${ }^{14}$. Uno de ellos es el de zonas rururbanas, que se refiere a zonas próximas a centros urbanos que experimentan un incremento residencial de población nueva, de origen principalmente ciudadano (Dezert et al., 1991: 7). Esta nueva configuración social del espacio rural pone en tela de juicio que, en la sociedad contemporánea, el hábitat continúe explicando el tipo de consumo. Es lo que ocurre en la práctica. En Cataluña el hábitat ya no es una variable estadísticamente significativa del tipo de consumo y en Andalucía explica muy poco (cuadro 2). Según este criterio, Cataluña se aproxima más a la tesis de la individuación que Andalucía. En esta última comunidad, continúan encontrándose diferencias entre las zonas rurales y urbanas, aunque la distancia que las separa se ha acortado notablemente.

Merece la pena analizar la asociación entre consumo y valores, empleando para ello la escala de materialismo y postmaterialismo (denominada también de supervivencia y autoexpresión). Los resultados de 1995 muestran la paradoja existente respecto a los valores postmaterialistas: solamente cuando se ha alcanzado cierto bienestar material, la población comienza a preocuparse por cuestiones postmateriales, como el medio ambiente, la emoción o la satisfacción en el trabajo. Como señalaba Maslow en su teoría de la motivación humana (1943), nunca desearemos componer música, decorar la casa o ir bien vestidos si nuestro estómago está vacío. Por esta razón, en el año 1995, cuanto más cerca se situaba la población de los valores postmaterialistas, mayor era el número de objetos de consumo que poseían por término medio. Las diferencias entre los grupos eran mayores en Andalucía que en Cataluña.

En el año 2006, en Andalucía, las personas más cercanas a los valores postmaterialistas siguen teniendo más objetos de consumo que los grupos anteriores, mientras que en Cataluña su consumo es menor. En Cataluña parece existir un porcentaje de postmaterialistas que, pese a disponer de bienestar material, renuncia a ciertos objetos materiales. Especialmente a las nuevas tecnologías, donde las diferencias con el grupo anterior se acrecientan. Hay un

13. Se consideran zonas rurales aquellas que alcanzan una población máxima de 10.000 habitantes; semiurbanas, de 10.000 a 100.000 habitantes, y urbanas, aquellas cuya población supera los 100.000 habitantes.

14. Para conocer más en detalle estas nuevas definiciones, ver J. Vicente (2003), «¿Nuevas palabras, nuevas ciudades?», Revista de Geografía, 2, 79-103. 
Cuadro 5. Porcentaje de población que dispone de los distintos objetos de consumo según la escala de valores materialistas y postmaterialistas, en Andalucía y Cataluña (2006)

\begin{tabular}{|c|c|c|c|c|c|c|c|c|c|c|}
\hline \multirow{2}{*}{$\begin{array}{l}\text { Objetos } \\
\text { de consumo }\end{array}$} & \multicolumn{5}{|c|}{ Andalucía } & \multicolumn{5}{|c|}{ Cataluña } \\
\hline & 1 & 2 & 3 & 4 & 5 & 1 & 2 & 3 & 4 & 5 \\
\hline Vivienda & 77,5 & 79,4 & 85,2 & 91,3 & 80,8 & 91 & 83,2 & 87,6 & 94,4 & 88,5 \\
\hline Automóvil & 66,6 & 74,7 & 72,7 & 75 & 76,9 & 61,9 & 82,8 & 85 & 83,5 & 84,6 \\
\hline Móvil & 69,6 & 79,3 & 83 & 82,6 & 96,2 & 69 & 88 & 92,6 & 96,6 & 88,5 \\
\hline DVD & 60,5 & 68,5 & 68,9 & 76,1 & 76,9 & 71,5 & 82,2 & 88,8 & 94,4 & 88,5 \\
\hline $\begin{array}{l}\text { Equipo } \\
\text { de sonido }\end{array}$ & 49 & 56,4 & 57,1 & 64,1 & 73,1 & 79,3 & 77,0 & 87,2 & 95,1 & 88,5 \\
\hline Ordenador & 30 & 41,3 & 44,9 & 48,9 & 84,6 & 31,6 & 49,4 & 58,2 & 78,3 & 73,1 \\
\hline Lavavajillas & 23,5 & 31,4 & 37,1 & 33,7 & 34,6 & 21,1 & 25,2 & 33,4 & 30,7 & 42,3 \\
\hline Media & 53,8 & 61,6 & 64,1 & 67,4 & 74,7 & 60,8 & 69,7 & 76,1 & 81,9 & 79,1 \\
\hline
\end{tabular}

Fuente: elaboración propia a partir del sondeo de opinión pública de ASEP (1995-2006).

menor porcentaje de ellos que posee vivienda propia, móvil, DVD, equipo de sonido y ordenador. La única excepción la constituye el lavavajillas, cuyo consumo es mayor. A tenor de los resultados obtenidos, pensamos si podría ser cierto que, tras estos datos, se encuentra el nuevo consumidor, informado y crítico, que, por poner un ejemplo, considera el lavavajillas como un objeto de consumo indispensable, puesto que ahorra energía y reduce el consumo de agua.

\subsubsection{El indice de posición social}

A modo de resumen de las variables analizadas, se presenta el «índice de posición social» ${ }^{15}$. En 1995, al mejorar la posición social, aumentaba el número medio de objetos materiales entre la población. En la sociedad andaluza, la posición social ocupaba un lugar importante como variable explicativa del acceso a los bienes de consumo, mientras que en Cataluña las diferencias eran menos acusadas. En el año 2006, contrariamente a lo que pudiéramos imaginar, en Andalucía las diferencias entre las posiciones más bajas y más altas siguen aumentando. En Cataluña ha ocurrido algo similar salvo en los dos últimos grupos de mayor estatus, donde el consumo de bienes materiales es

15. Para Galtung, el centro social es el conjunto de posiciones sociales mejor recompensadas por la sociedad (incluyendo en el concepto de recompensas no sólo las económicas, sino también las de prestigio y poder, siguiendo una tradición ya secular en el pensamiento sociológico), mientras que la periferia social sería lo contrario, es decir, el conjunto de posiciones sociales peor recompensadas (incluso rechazadas) por la sociedad. El índice de posición social se obtiene de la combinación de diferentes variables: sexo, edad, ocupación del entrevistado, educación del entrevistado, provincia de residencia, hábitat de residencia y nivel de ingresos en el hogar. 
Cuadro 6. Media de objetos de consumo según el índice de posición social en Andalucía y Cataluña (1995-2006)

\begin{tabular}{|c|c|c|c|c|c|c|c|c|}
\hline \multirow{3}{*}{$\frac{\text { Posición social }}{0 . \text { Muy baja }}$} & \multicolumn{4}{|l|}{1995} & \multicolumn{4}{|l|}{2006} \\
\hline & \multicolumn{2}{|c|}{ Andalucía } & \multicolumn{2}{|c|}{ Cataluña } & \multicolumn{2}{|c|}{ Andalucía } & \multicolumn{2}{|c|}{ Cataluña } \\
\hline & 1,90 & 100 & 2,45 & 100 & 2,86 & 100,0 & 4,3 & 100,0 \\
\hline 1. & 2,14 & 112,6 & 2,50 & 102,0 & 3,69 & 129,0 & 4,8 & 111,1 \\
\hline 2. & 2,53 & 133,1 & 2,71 & 110,6 & 4,44 & 155,2 & 5,5 & 125,8 \\
\hline 3. & 2,61 & 137,4 & 2,97 & 121,2 & 5,21 & 182,3 & 6,4 & 146,7 \\
\hline 4. & 2,96 & 155,8 & 3,18 & 129,8 & 5,55 & 194,2 & 6,7 & 154,9 \\
\hline 5. & 3,08 & 162,1 & 3,42 & 139,6 & 5,68 & 198,6 & 7,2 & 165,4 \\
\hline 6. & 3,53 & 185,8 & 3,57 & 145,7 & 6,07 & 212,3 & 7,8 & 178,7 \\
\hline 7. & 3,86 & 203,1 & 3,95 & 161,2 & 6,96 & 243,4 & 7,5 & 173,8 \\
\hline 8. Muy alta & 4,64 & 244,2 & 4,39 & 179,2 & $8,10^{*}$ & 283,2 & $7,0^{*}$ & 161,2 \\
\hline Eta cuadrado y $\mathrm{N}$ & 0,108 & 2.385 & 0,101 & 2.094 & 0,126 & 2.297 & 0,149 & 2.123 \\
\hline
\end{tabular}

Fuente: elaboración propia a partir del sondeo de opinión pública de ASEP (1995-2006).

* Los resultados sombreados tienen una muestra inferior a veinte casos y hay que ser precavidos con su interpretación.

menor que en el año 1995 y menor que el del grupo anterior. Parece cumplirse la hipótesis de Galtung: «la posición social puede explicar el postmaterialismo. Las personas cerca del centro social, al ser las iniciadoras de nuevos valores sociales, son las que adoptan la orientación postmaterialista como respuesta adaptativa ante la situación actual, caracterizada por un creciente bienestar material acompañado de crecientes amenazas al deterioro del medio ambiente» (Díez Nicolás, 1992: 21-35). Se podría interpretar que los valores de autoexpresión están provocando un cambio de actitudes hacia el consumo en los grupos con mejor posición social en Cataluña, algo que, sin duda, aún no se aprecia en Andalucía.

\section{Conclusiones}

Se exponen los resultados de las hipótesis planteadas inicialmente en relación con la teoría de la modernización:

- Hipótesis 1: Andalucía está más cerca de Cataluña en el año 2006 que en 1995. Los datos rechazan esta hipótesis. Andalucía sigue la tendencia de Cataluña, pero, como Cataluña sigue avanzando, las diferencias entre ambas comunidades persisten. Según el indicador empleado, Andalucía está más lejos de Cataluña en 2006 que en 1995.

- Hipótesis 2: La posesión de objetos de consumo es mayor en Cataluña que en Andalucía. Cataluña poseía y posee más objetos de consumo que Andalucía. Estas diferencias son especialmente notorias en las tecnologías. 
- Hipótesis 3: Hay una mayor posesión de objetos de consumo en 2006 que en 1995. Hay una mayor extensión de los objetos de consumo entre la población (cuadro 1).

- Hipótesis 4: Hay mayor igualdad social respecto al consumo en 2006 que en 1995. La comparación entre 1995 y 2006 desvela que, en la mayoría de las variables analizadas, hay una mayor desigualdad entre unos grupos y otros. Así ocurre con el nivel educativo, la posición social, la edad y la escala de valores en Andalucía y Cataluña. El nivel de ingresos muestra una mayor desigualdad en Andalucía, mientras que da a conocer una mayor igualdad en Cataluña. No obstante, esta variable ofrece problemas muestrales, de modo que su interpretación en Cataluña hay que realizarla con precaución. Solamente el hábitat y la ocupación, es decir, las variables que menos explican, muestran una mayor igualdad entre los grupos con el paso del tiempo.

- Hipótesis 5: Hay mayor igualdad social en Cataluña que en Andalucía. En Cataluña, las diferencias entre los grupos son menores. Lo que viene a decir que en Cataluña hay una mayor igualdad entre los grupos según ingresos, educación, edad, posición social, ocupación, valores y hábitat, que en Andalucía. Por esta cuestión se diría que, aunque han avanzado hacia una desigualdad en el periodo 1995-2006, en 2006 Cataluña sigue siendo una sociedad en la que el consumo presenta menos diferencias entre los extremos.

- Hipótesis 6: Las categorías sociales explican menos en 2006 que en 1995. El análisis de varianza muestra que las diferencias entre las variables y el consumo siguen siendo significativas, con la excepción del hábitat. El lugar de residencia ya no explica la cantidad de objetos de consumo en Cataluña, y en Andalucía la asociación es muy débil. Con el resto de variables, la asociación (eta) no es muy fuerte, pero tampoco lo era en 1995. Las variables que más explican el consumo siguen siendo el nivel de ingresos y el nivel educativo. El nivel de ingresos cada vez explica menos y el estatus ocupacional tiene poca influencia en la sociedad postmoderna. Por el contrario, la edad y los valores individuales explican cada vez más el consumo, aunque estos últimos tienen aún poca influencia.

- Hipótesis 7: Las categorías sociales explican menos en Cataluña que en Andalucía. En el año 1995, las categorías sociales explicaban más en Andalucía que en Cataluña, pero en el año 2006 la relación se invierte, con la excepción del hábitat. El nivel educativo cada vez explica menos el consumo en Andalucía, mientras que en Cataluña se ha convertido en la variable que más explica. Razón que puede deberse a la mayor extensión de las nuevas tecnologías, en las que el nivel educativo desempeña un gran papel.

- Hipótesis 8 y 9: Es posible encontrar un nuevo consumidor que, teniendo posibilidades económicas y educativas, consuma menos objetos. El nuevo consumidor será localizado en los grupos con mejor posición social. Se percibe cierta aleatoriedad en el comportamiento y algunas pautas de menor consumo donde creemos reconocer al nuevo consumidor. Entre los grupos con mejor 
posición social y valores postmaterialistas, parece estar descendiendo el consumo de ciertos objetos materiales, lo que podría implicar la preferencia en la elección de otras actividades no materiales que les concediesen una mayor satisfacción personal. Esta tendencia se percibe en Cataluña y no en Andalucía, donde persiste la posibilidad de combinar los valores postmaterialistas con el consumo de bienes materiales.

\section{Referencias bibliográficas}

AlONSO, L.E. y CONDE, F. (1994). Historia del consumo en España: Una aproximación a sus orígenes y primer desarrollo. Madrid: Debate.

Alonso, L.E. (2005). La era del consumo. Madrid: Siglo XXI.

ASEP (1995, 2006). La opinión pública de los españoles. [www.jdsurvey.net]

BAUdrillard, J. (1997). The consumer society. Londres: Sage Publications.

BAUMAN, Z. (1992). Intimations of postmodernity. Nueva York: Routledge.

BECK, U. (1992). Risk society: Towards a new modernity. Londres: Sage Publications. BELL, D. (1989). El advenimiento de la sociedad post-industrial. Madrid: Alianza.

BERICAT, E. (2003). «Fragmentos de la realidad social posmoderna». REIS, 102, 9-46.

BERICAT, E. y MARTíN-LAGOS, M.D. (2006). «La transformación de los hogares españoles y andaluces». Realidad Social, 3. Sevilla: Centro de Estudios Andaluces.

Bericat, E. y MenChÓN, A. (2006). «La brecha digital en Andalucía». Actualidad, 7. Sevilla: Centro de Estudios Andaluces.

Bocock, R. (1995). El consumo. Madrid: Talasa.

Bourdieu, P. (1988). La distinción: criterios y bases sociales del gusto. Madrid: Taurus.

BoYD, M. (2000). Between Mecca and Beijing: Modernization and consumption among urban Chinese muslims. Stanford: Stanford University Press.

CAïs, J. (2001). "Cambio social y desigualdades en los países mediterráneos». REIS, 94, 37-78.

CAMPBELL, C. (1998): «Consumption and the rhetorics of need and want». Journal of Design History, 11 (3), 235-246.

CASTILlO, J. (1969). "Consideraciones en torno a la sociedad de consumo de masas», en Miguez, A. et al., España, ¿una sociedad de consumo? Madrid: Guadiana, 29-53.

- (1982). «Los hijos de la sociedad de consumo». REIS, 17, 39-51.

De Miguel, A. (1969). «Clase social y consumo en España», en Miguez, A. et al., España, ¿una sociedad de consumo? Madrid: Guadiana, 55-83.

- (1987). Ahora mismo: Sociología de la vida cotidiana. Madrid: Espasa-Calpe.

- (2001). La vida cotidiana de los españoles en el siglo XXI. Barcelona: Planeta.

- (2002). Los pecados capitales de los españoles y el consumo. Madrid: Instituto de Estudios Económicos.

De Miguel, J. (1998). Estructura y cambio social en España. Madrid: Alianza.

DezerT, B. et al. (1991). La périurbanisation en France. París: Sedes.

DÍEZ, J. (1992). «Posición social, información y postmaterialismo». REIS, 57, 21-35.

Featherstone, M. (1991). Consumer culture and postmodernism. Londres: Sage Publications.

FLORIDA, R. (2002). The creative class. Nueva York: Basic Books.

GALTUNG, J. (1964). «Foreign policy opinion as a function of social position». Journal of Peace Research, 3-4. Oslo. 
Gazeboom, H. y Treiman, D.J. (2007). "Ascription and achievement in occupational attainment in comparative perspective». The Sixth Meeting of the Russell Sage Foundation/Carnegie Corporation.

GidDENS, A. (1990). The consequences of modernity. Stanford: Stanford University Press.

- (1991). Modernity and self-identity: Self and society in the late modern age. Stanford: Stanford University Press.

Gobernado, R. (1996). Análisis comparado de las estructuras sociales de Andalucia y Cataluña. Málaga: Universidades de Málaga y Almería.

GoldTHORPE, John H.; LOCKWOOD, David; BECHHOFER, Frank y PLATT, Jennifer (1968). The affluent worker: political attitudes and behaviour. Cambridge University Press.

Hamilton, G.G. (1977). "Chinese consumption of foreign commodities: A comparative perspective». American Sociological Review, 42 (6), diciembre, 877-891.

HuBER, J. (2000). «Towards industrial ecology: Sustainable development as a concept of ecological modernization». Journal of Environmental Policy and Planning, 2.

INGLEHART, R. (1999). Modernización y posmodernización: el cambio cultural, económico y político en 43 sociedades. Madrid: Centro de Investigaciones Sociológicas.

INGLEHART, R. y WELZEL, C. (2006). Modernización, cambio cultural y democracia: La secuencia del desarrollo humano. Madrid: Centro de Investigaciones Sociológicas.

Klein, N. (2001). No logo: El poder de las marcas. Barcelona: Paidós.

Maslow, A. (1943). "A theory of human motivation». Psycological Review, 50, 370-396. Míguez, A. et al. (1969). España, ¿Una sociedad de consumo? Madrid: Guadiana.

Oltra, C. (2005). «Modernización ecológica y sociedad del riesgo». Papers, 78, 133 149.

ORTí, A. (1994). «La estrategia de la oferta en la sociedad neocapitalista de consumo: génesis y praxis de la investigación motivacional». Política y Sociedad, 16, 37-92. Universidad Complutense de Madrid.

RAMíreZ, A. et al. (2002). «Consumerismo y movimiento de los consumidores». REIS, 99, 145-176.

Rostov, W.W. (1993). Las etapas del crecimiento económico. Madrid: Ministerio de Trabajo y Seguridad Social.

SCHNAIBERG, A. (1970): "Measuring modernism: Theoretical and empirical explorations». The American Journal of Sociology, 76 (3), noviembre, 399-425. The University of Chicago Press.

Simmel, G. (2002). Cuestiones fundamentales de sociología. Barcelona: Gedisa.

TEZANOS, J.F. (1984). «Cambio social y modernización en la España actual». REIS, 28, 19-62.

Veblen, T. (1963). Teoría de la clase ociosa. México: Fondo de Cultura Económica.

WALLERSTEIN, I. (1979). El moderno sistema mundial [I]: La agricultura capitalista y los orígenes de la economía-mundo europea en el siglo XVI. Madrid: Siglo XXI.

Weber, M. (1955). La ética protestante y el espíritu del capitalismo. Madrid: Revista de Derecho Privado.

YATES, R. (2002): «A brief history of British drug policy, 1950-2001». Drugs: Education, Prevention and Policy, 9 (2), 113-124. 